\title{
Perioperative Management of Patients With Ankylosing Spondylitis Undergoing Spine Surgery
}

\author{
Arman Zakaryan ${ }^{1 *}$ and Knarik Ginosyan ${ }^{2}$ \\ ${ }^{1}$ Department of Neurosurgery, Yerevan State Medical University after Mkhitar Heratsi, Yerevan, Armenia, ${ }^{2}$ Department of \\ Rheumatology, Yerevan State Medical University after Mkhitar Heratsi, Yerevan, Armenia
}

Keywords: ankylosing spondylitis, spine surgery, spine deformity, perioperative management

\section{OPEN ACCESS}

Edited by:

Suren Soghomonyan, The Ohio State University,

United States

Reviewed by:

Gurgen Harutyunyan, Hospital 9 de Octubre, VITHAS, Spain

*Correspondence:

Arman Zakaryan

armzak@gmail.com

Specialty section: This article was submitted to Cardiovascular and Smooth Muscle Pharmacology, a section of the journal Frontiers in Pharmacology

Received: 26 April 2020 Accepted: 23 June 2020 Published: 09 July 2020

Citation: Zakaryan A and Ginosyan K (2020) Perioperative Management of Patients With Ankylosing Spondylitis Undergoing Spine Surgery. Front. Pharmacol. 11:1017. doi: 10.3389/fphar.2020.01017
Ankylosing spondylitis (AS) is the most frequent type of seronegative spondyloarthropathy, which mainly involves the axial spine. In advanced cases, the chronic inflammatory process can cause fibrosis and calcification, which leads to loss of flexibility and fusion of the vertebrae, resembling a "bamboo", with a fixed posture, generally known as chin-on-chest deformation.

The functional limitations of this particular deformity are significant: the patients are unable to look forward, make visual contact, and have difficulty eating. All these factors with typically concomitant osteoporosis will significantly increase the risk of spinal injury with severe instability, deformity, and, most importantly, deteriorating neurological function or paralysis requiring spinal surgery. Common indications for spinal intervention on patients with AS are:

- Uncontrolled, severe and continuous back and neck pain

- Deteriorating neurological deficit caused by spinal deformity

- Unstable spinal fracture

- Inability to hold the head up and see horizontally

- Serious difficulties in eating and drinking because of spinal deformity.

Spinal surgery on these patients is related to a greater risk of surgical and anesthesia-related complications-such as difficult intubation with necessity of awake fiberoptic intubation, infection, need for blood transfusions, respiratory and cardiac problems, and renal dysfunction postoperatively (Puvanesarajah et al., 2017). Therefore, assessment of medical comorbidities, associated with both the disease and the treatment of AS is an important component of the medical clearance before surgery.

Cardiovascular Concerns: According to the American Heart Association and American College of Cardiology (ACC/AHA) cardiac risk assessing guidelines in preoperative surgical preparation of patients with AS, cardiovascular disease is advanced if functional capacity is not reached to a minimum of four Metabolic Equivalents (METS) (Fleisher et al., 2014). These patients with poor functional capacity (<4 METS) are at greater cardiac risk (Goodman and Bass, 2018).

Pulmonary Concerns: Significant spinal deformity in AS can result in restrictive respiratory physiology, with notable decreases in vital lung capacity (Berdal et al., 2012). This may make ventilation during surgery challenging, especially when a prone position is anticipated. For those patients, aggressive pulmonary hygiene in the postoperative period can be encouraged

Neuromuscular Concerns: A thorough physical examination should be performed to document any preoperative sensory or motor deficits, especially in patients undergoing spinal surgery. 
Notation of any motor weakness is important as this may affect the plan for intraoperative neuromonitoring and the anesthesia (Sciubba et al., 2008).

Renal Concerns: Since AS patients receive non-steroidal antiinflammatory drugs (NSAID) for a long period, interstitial nephritis may be present due to NSAID use. Advanced AS may be associated chronic kidney dysfunction due to amyloidosis, IgA-nephropathy, and tubulointerstitial nephritis (Ye et al., 2019).

Bone loss Concerns: Taking into consideration that osteoporosis is a common complication of AS, with an incidence between $18.7 \%$ and $62 \%$, all patients undergoing spinal surgery should be treated before and after surgery for osteoporosis (van der Weijden et al., 2012).

According to the Assessment of SpondyloArthritis International Society/European League Against Rheumatism (ASAS-EULAR) management suggestions for AS, NSAIDs are considered the firstline drug treatment. To prevent further progression of the disease and achieve remission with improved quality of life, diseasemodifying antirheumatic drugs (DMARD) are required: synthetic (such as sulfasalazine) and biological (anti-TNF- $\alpha$ agents such as infliximab, etanercept, adalimumab, golimumab, etc. as well as inhibitors of IL-17-secukinumab) (van der Heijde et al., 2017).

Taking into account the need for chronic therapy and associated side effects of those drugs, patients undergoing surgery need dosage adjustment.

NSAIDs: The perioperative use of NSAIDs or aspirin may be associated with bleeding complications. This is because NSAIDinduced inhibition of COX-1 with reduction of thromboxane A2, which, in turn, will decrease vasoconstriction and platelet aggregation and increase the bleeding time. This effect is reversed only after drug withdrawal. Considering the average half-life of an NSAID, they must be withdrawn at some point prior to surgery. (Franco et al., 2017). On the other hand, in patients, whose cardiovascular risk exceeds the intraoperative benefit (for example, patients with cardiac stents), it's recommended to continue the aspirin during the perioperative period (Oscarsson et al., 2010; Gerstein et al., 2015). In such situations, the surgeon must be alerted and prepared for bleeding during surgery, even though cardiac doses of aspirin, in general, are not associated with

\section{REFERENCES}

Berdal, G., Halvorsen, S., van der Heijde, D., Mowe, M., and Dagfinrud, H. (2012). Restrictive pulmonary function is more prevalent in patients with ankylosing spondylitis than in matched population controls and is associated with impaired spinal mobility: a comparative study. Arthritis Res. Ther. 14 (1), R19. doi: 10.1186/ar3699

Fleisher, L. A., Fleischmann, K. E., Auerbach, A. D., Barnason, S. A., Beckman, J. A., Bozkurt, B., et al. (2014). 2014 ACC/AHA guideline on perioperative cardiovascular evaluation and management of patients undergoing noncardiac surgery: a report of the American College of Cardiology/American Heart Association Task Force on practice guidelines. J. Am. Coll. Cardiol. 64 (22), e77-137. doi: 10.1016/j.jacc.2014.07.944

Franco, A. S., Iuamoto, L. R., and Pereira, R. M. R. (2017). Perioperative management of drugs commonly used in patients with rheumatic diseases: a review. Clinics 72 (6), 386-390. doi: 10.6061/clinics/2017(06)09 a significant risk of bleeding (Vetter et al., 2014). Since COX-2 inhibitors have little effects on platelet function, there is no need to suspend them during the perioperative period. (Leese et al., 2000; Franco et al., 2017). However, this group of drugs is associated with the risk of significant cardiovascular adverse outcomes.

Glucocorticosteroids (GCS): Patients continuously taking GCS are at risk of intraoperative hemodynamic instability and postoperative infection. A few small randomized controlled trials and systematic reviews demonstrate that there are no significant hemodynamic changes between patients receiving a daily dose of GCS compared to those receiving "stress-dose steroids" preoperatively. (Goodman et al., 2017). Therefore it's recommended to use the usual daily dose of GCS or prednisone less than $20 \mathrm{mg} /$ day, rather than the "stress dose" with regards to infection risk (Somayaji et al., 2013).

DMARDs: Non-biologic synthetic DMARDs are recommended to continue at the time of surgery since it was shown that the risk of infections is not increased. Perioperative continuation of DMARDS also decreases the risk of exacerbation of the main disease after the surgery (Goodman et al., 2017).

Systematic reviews and meta-analyses of biologic DMARDs revealed an increased risk of serious infections. Most commonly the respiratory and urinary tract infections, skin, and opportunistic infections are developed, including hepatitis B and C, tuberculosis, and various fungal infections such as histoplasmosis (Nard et al., 2015).

Therefore, all current biologic DMARDs should be withdrawn before the surgery and it is important to schedule the procedure after the active period of these drugs (Goodman et al., 2017).

In conclusion, proper perioperative management, consisting of preoperative patient planning, as well as intraoperative and postoperative patient monitoring and care, are required. Further investigations are needed to build up a reliable, evidence-based strategy of effective perioperative management of AS patients undergoing spine surgery.

\section{AUTHOR CONTRIBUTIONS}

$\mathrm{AZ}$ and $\mathrm{KG}$ contributed to the writing of the manuscript.

Gerstein, N. S., Carey, M. C., Cigarroa, J. E., and Schulman, P. M. (2015). Perioperative aspirin management after POISE-2: some answers, but questions remain. Anesth. Analg. 120 (3), 570-575. doi: 10.1213/ANE.0000000000000589

Goodman, S. M., and Bass, A. R. (2018). Perioperative medical management for patients with RA, SPA, and SLE undergoing total hip and total knee replacement: a narrative review. BMC Rheumatol. 2, 1-8. doi: 10.1186/s41927-018-0008-9

Goodman, S. M., Springer, B., Guyatt, G., Abdel, M. P., Dasa, V., George, M., et al. (2017). American College of Rheumatology/American Association of Hip and Knee Surgeons Guideline for the Perioperative Management of Antirheumatic Medication in Patients With Rheumatic Diseases Undergoing Elective Total Hip or Total Knee Arthroplasty. Arthritis Care Res. 69 (8), 1111-1124. doi: 10.1002/ acr. 23274

Leese, P. T., Hubbard, R. C., Karim, A., Isakson, P. C., Yu, S. S., and Geis, G. S. (2000). Effects of celecoxib, a novel cyclooxygenase-2 inhibitor, on platelet function in healthy adults: a randomized, controlled trial. J. Clin. Pharmacol. 40 (2), 124-132. doi: 10.1177/00912700022008766 
Nard, F. D., Todoerti, M., Grosso, V., Monti, S., Breda, S., Rossi, S., et al. (2015). Risk of hepatitis $\mathrm{B}$ virus reactivation in rheumatoid arthritis patients undergoing biologic treatment: Extending perspective from old to newer drugs. World J. Hepatol. 7 (3), 344-361. doi: 10.4254/wjh.v7.i3.344

Oscarsson, A., Gupta, A., Fredrikson, M., Jarhult, J., Nystrom, M., Pettersson, E., et al. (2010). To continue or discontinue aspirin in the perioperative period: a randomized, controlled clinical trial. Br. J. Anaesth. 104 (3), 305-312. doi: 10.1093/bja/aeq003

Puvanesarajah, V., Cancienne, J. M., Shimer, A. L., Shen, F. H., and Hassanzadeh, H. (2017). Complications after Fusion for Thoracolumbar Fractures in Patients with Ankylosing Spondylitis. Global Spine J. 7 (1), 28-32. doi: 10.1055/s-00361583175

Sciubba, D. M., Nelson, C., Hsieh, P., Gokaslan, Z. L., Ondra, S., and Bydon, A. (2008). Perioperative challenges in the surgical management of ankylosing spondylitis. Neurosurg. Focus 24 (1), E10. doi: 10.3171/FOC/2008/24/1/E10

Somayaji, R., Barnabe, C., and Martin, L. (2013). Risk factors for infection following total joint arthroplasty in rheumatoid arthritis. Open Rheumatol. J. 7, 119-124. doi: 10.2174/1874312920131210005

van der Heijde, D., Ramiro, S., Landewe, R., Baraliakos, X., Van den Bosch, F., Sepriano, A., et al. (2017). update of the ASAS-EULAR management recommendations for axial spondyloarthritis. Ann. Rheum. Dis. 76 (6), 978991. doi: 10.1136/annrheumdis-2016-210770 van der Weijden, M. A., Claushuis, T. A., Nazari, T., Lems, W. F., Dijkmans, B. A., and van der Horst-Bruinsma, I. E. (2012). High prevalence of low bone mineral density in patients within 10 years of onset of ankylosing spondylitis: a systematic review. Clin. Rheumatol. 31 (11), 1529-1535. doi: 10.1007/ s10067-012-2018-0

Vetter, T. R., Short, R., Hawn, M. T., and Marques, M. B. (2014). Perioperative management of the patient with a coronary artery stent. Anesthesiology 121 (5), 1093-1098. doi: 10.1097/ALN.0000000000000451

Ye, W., Zhuang, J., Yu, Y., Li, H., Leng, X., Qian, J., et al. (2019). Gender and chronic kidney disease in ankylosing spondylitis: a single-center retrospectively study. BMC Nephrol. 20 (1), 457. doi: 10.1186/s12882-019-1658-6

Conflict of Interest: The authors declare that the research was conducted in the absence of any commercial or financial relationships that could be construed as a potential conflict of interest.

Copyright (c) 2020 Zakaryan and Ginosyan. This is an open-access article distributed under the terms of the Creative Commons Attribution License (CC BY). The use, distribution or reproduction in other forums is permitted, provided the original author(s) and the copyright owner(s) are credited and that the original publication in this journal is cited, in accordance with accepted academic practice. No use, distribution or reproduction is permitted which does not comply with these terms. 